Bull. Korean Math. Soc. 50 (2013), No. 6, pp. 2027-2034

http://dx.doi.org/10.4134/BKMS.2013.50.6.2027

\title{
ADDITIVITY OF JORDAN TRIPLE PRODUCT HOMOMORPHISMS ON GENERALIZED MATRIX ALGEBRAS
}

\author{
Sang OG Kim and Choonkil Park
}

\begin{abstract}
In this article, it is proved that under some conditions every bijective Jordan triple product homomorphism from generalized matrix algebras onto rings is additive. As a corollary, we obtain that every bijective Jordan triple product homomorphism from $M_{n}(\mathcal{A})(\mathcal{A}$ is not necessarily a prime algebra) onto an arbitrary ring $\mathcal{R}^{\prime}$ is additive.
\end{abstract}

\section{Introduction and statements of the results}

Let $\mathcal{R}$ be a commutative ring with identity, $\mathcal{A}$ and $\mathcal{B}$ be two associative algebras over $\mathcal{R}$. Let $\mathcal{M}$ be an $(\mathcal{A}, \mathcal{B})$-bimodule and $\mathcal{N}$ a $(\mathcal{B}, \mathcal{A})$-bimodule. Assume that there are two bimodule homomorphisms $\varphi: \mathcal{M} \otimes_{\mathcal{B}} \mathcal{N} \rightarrow \mathcal{A}$ and $\psi: \mathcal{N} \otimes_{\mathcal{A}} \mathcal{M} \rightarrow \mathcal{B}$ satisfying the associativity conditions: $(M N) M^{\prime}=M\left(N M^{\prime}\right)$ and $(N M) N^{\prime}=N\left(M N^{\prime}\right)$ for all $M, M^{\prime} \in \mathcal{M}$ and $N, N^{\prime} \in \mathcal{N}$, where we put $M N=\varphi(M \otimes N)$ and $N M=\psi(N \otimes M)$. A generalized matrix algebra $\operatorname{Mat}(\mathcal{A}, \mathcal{M}, \mathcal{N}, \mathcal{B})$ is an associative algebra of the form

$$
\operatorname{Mat}(\mathcal{A}, \mathcal{M}, \mathcal{N}, \mathcal{B})=\left\{\left[\begin{array}{cc}
A & M \\
N & B
\end{array}\right]: A \in \mathcal{A}, M \in \mathcal{M}, N \in \mathcal{N}, B \in \mathcal{B}\right\}
$$

under the usual matrix-like multiplication, where at least one of the two bimodules $\mathcal{M}$ and $\mathcal{N}$ is distinct from zero. In the above definition of generalized matrix algebras, if $\mathcal{M}$ is faithful as a left $\mathcal{A}$-module and also as a right $\mathcal{B}$-module and $\mathcal{N}=\{0\}$, then the associative $\mathcal{R}$-algebra

$$
\operatorname{Tri}(\mathcal{A}, \mathcal{M}, \mathcal{B})=\left\{\left[\begin{array}{cc}
A & M \\
0 & B
\end{array}\right]: A \in \mathcal{A}, M \in \mathcal{M}, B \in \mathcal{B}\right\}
$$

is usually called a triangular algebra. The most important examples of triangular algebras are upper triangular matrix algebras, block upper triangular matrix algebras and nest algebras. Obviously, the triangular algebras and $M_{n}(\mathcal{A})$, the

Received November 6, 2012; Revised April 24, 2013.

2010 Mathematics Subject Classification. Primary 16W99, 47B47 47L35.

Key words and phrases. Jordan triple product homomorphism, generalized matrix algebra, additive map. 
matrix algebra over $\mathcal{A}$ are kinds of generalized matrix algebras. We refer the reader to [22] for some classical examples of generalized matrix algebras.

Let $\phi$ be a map from $\mathcal{A}$ to $\mathcal{B}$ and $A, B, C$ be arbitrary elements of $\mathcal{A}$.

(1) $\phi$ is said to be multiplicative if

$$
\phi(A B)=\phi(A) \phi(B) .
$$

(2) $\phi$ is called a Jordan map if

$$
\phi(A B+B A)=\phi(A) \phi(B)+\phi(B) \phi(A) .
$$

(3) $\phi$ is called a Jordan triple map if

$$
\phi(A B C+C B A)=\phi(A) \phi(B) \phi(C)+\phi(C) \phi(B) \phi(A) .
$$

(4) $\phi$ is said to be a Jordan triple product homomorphism if

$$
\phi(A B A)=\phi(A) \phi(B) \phi(A) .
$$

In studying preservers on algebras or rings, one usually assumes additivity in advance. Recently, however, a growing number of papers began investigating preservers that are not necessarily additive. Characterizing the interrelation between the multiplicative and additive structures of a ring or algebra is an interesting topic. This question was first studied by Martindale [18] who showed the surprising result that every bijective multiplicative map from a prime ring containing a nontrivial idempotent onto an arbitrary ring is necessarily additive. For operator algebras, the same problem was treated in $[1,14,21]$. In the papers $[2,3,7,12,13,15,19]$, the additivity of maps on operator algebras which are multiplicative with respect to other products, such as the Jordan product, the Jordan triple product or Jordan triple product homomorphisms were investigated. Also, the papers $[6,9,20]$ studied the similar questions for elementary maps and Jordan elementary maps on rings or operator algebras.

Ling and $\mathrm{Lu}$ [11] studied Jordan maps of nest algebras, a kind of triangular algebras coming from operator theory. They showed that every Jordan bijective map on a standard subalgebra of a nest algebra is additive. This result was extended by Ji [4] to Jordan surjective map pair of triangular algebras. Cheng and Jing [1] proved that every multiplicative bijective map, Jordan bijective map, Jordan triple bijective map and elementary surjective map on triangular algebras is additive. Recently, Li and Xiao [10] extended the results of Ji [4] to generalized matrix algebras. In fact, they proved that every multiplicative bijective map, Jordan bijective map, Jordan triple bijective map on a generalized matrix algebra is additive under some conditions.

For the Jordan triple product homomorphisms, Li and Jing [8] showed that if $\mathcal{R}$ is a 2 -torsion free prime ring containing a nontrivial idempotent and $\mathcal{R}^{\prime}$ is an arbitrary ring, then every bijective Jordan triple product homomorphism is additive. Kuzma [7] described the forms of Jordan triple product homomorphisms on matrix algebras and Molnár [19] obtained the exact forms of Jordan triple product homomorphisms between standard operator algebras. 
There is a connection between Jordan triple product homomorphisms and Lie triple product homomorphisms, the $\mathcal{R}$-linear maps $\phi: \mathcal{A} \rightarrow \mathcal{B}$ such that for every $A, B, C \in \mathcal{A}, \phi([[A, B], C])=[[\phi(A), \phi(B)], \phi(C)]$, where $[A, B]=$ $A B-B A$ is the Lie multiplication. In recent years, there has been some interest in studying Lie triple isomorphisms and Lie triple derivations. We refer the interested readers to $[5,16,17,23]$.

Motivated by the results of [8] and [10], we prove that under some conditions every bijective Jordan triple product homomorphism from generalized matrix algebras onto rings is additive. As a byproduct, we obtain that a bijective Jordan triple product homomorphism from $M_{n}(\mathcal{A})$ onto an arbitrary ring $\mathcal{R}^{\prime}$ is additive, where $\mathcal{A}$ is not necessarily a prime algebra. We mention that we use some techniques similar to those of [12].

Throughout this article, let $\mathcal{G}$ be a generalized matrix algebra $\operatorname{Mat}(\mathcal{A}, \mathcal{M}, \mathcal{N}$, $\mathcal{B})$. For convenience, we set

$$
\begin{aligned}
& \mathcal{G}_{11}=\left\{\left[\begin{array}{cc}
A & 0 \\
0 & 0
\end{array}\right]: A \in \mathcal{A}\right\} ; \mathcal{G}_{12}=\left\{\left[\begin{array}{cc}
0 & M \\
0 & 0
\end{array}\right]: M \in \mathcal{M}\right\} \\
& \mathcal{G}_{21}=\left\{\left[\begin{array}{cc}
0 & 0 \\
N & 0
\end{array}\right]: N \in \mathcal{N}\right\} ; \mathcal{G}_{22}=\left\{\left[\begin{array}{cc}
0 & 0 \\
0 & B
\end{array}\right]: B \in \mathcal{B}\right\} .
\end{aligned}
$$

Then every element $A \in \mathcal{G}$ can be written as $A=A_{11}+A_{12}+A_{21}+A_{22}$, where $A_{i j} \in \mathcal{G}_{i j}$ for $1 \leq i, j \leq 2$.

Our main result reads as follows.

Theorem 1.1. Let $\mathcal{R}$ be a commutative ring with identity, $\mathcal{A}$ and $\mathcal{B}$ be two unital algebras over $\mathcal{R}$. Let $\mathcal{G}$ be the generalized matrix algebra $\operatorname{Mat}(\mathcal{A}, \mathcal{M}, \mathcal{N}, \mathcal{B})$ which satisfies the following conditions:

(1) for $M \in \mathcal{M}, N M N=0$ for every $N \in \mathcal{N}$ implies $M=0$,

(2) for $N \in \mathcal{N}, M N M=0$ for every $M \in \mathcal{M}$ implies $N=0$,

(3) $\mathcal{M}$ is faithful as a left $\mathcal{A}$-module, that is, for $A \in \mathcal{A}, A M=0$ for every $M \in \mathcal{M}$ implies $A=0$,

(4) for $B \in \mathcal{B}, M B N=0$ for every $M \in \mathcal{M}$ and $N \in \mathcal{N}$ implies $B=0$.

Then every bijective Jordan triple product homomorphism $\phi$ from $\mathcal{G}$ onto an arbitrary ring $\mathcal{R}^{\prime}$ is additive.

It is clear $M_{n}(\mathcal{A})$ satisfies the conditions of Theorem 1 for (not necessarily prime) unital algebras $\mathcal{A}$. Hence we have the following corollary.

Corollary 1.2. Let $\mathcal{A}$ be a unital (not necessarily prime) algebra over a commutative ring $\mathcal{R}$. Then every bijective Jordan triple product homomorphism $\phi$ from $M_{n}(\mathcal{A})$ onto an arbitrary ring $\mathcal{R}^{\prime}$ is additive for $n \geq 2$.

\section{Proofs}

We prove the theorem in a series of lemmas.

Lemma 2.1. $\phi(0)=0$. 
Proof. Since $\phi$ is surjective there exists an element $A \in \mathcal{G}$ such that $\phi(A)=0$. Then $\phi(0)=\phi(0 A 0)=\phi(0) \phi(A) \phi(0)=0$.

Lemma 2.2. $\phi\left(A_{11}+A_{12}+A_{21}+A_{22}\right)=\phi\left(A_{11}\right)+\phi\left(A_{12}\right)+\phi\left(A_{21}\right)+\phi\left(A_{22}\right)$ for every $A_{i j} \in \mathcal{G}_{i j}$.

Proof. Let $S \in \mathcal{G}$ be an element such that $\phi(S)=\phi\left(A_{11}\right)+\phi\left(A_{12}\right)+\phi\left(A_{21}\right)+$ $\phi\left(A_{22}\right)$. Then we have for every $X_{i j}, 1 \leq i, j \leq 2$

$$
\begin{aligned}
\phi\left(X_{i j} S X_{i j}\right) & =\phi\left(X_{i j}\right) \phi(S) \phi\left(X_{i j}\right) \\
& =\phi\left(X_{i j}\right) \sum_{l, t} \phi\left(A_{l t}\right) \phi\left(X_{i j}\right) \\
& =\sum_{l, t} \phi\left(X_{i j} A_{l t} X_{i j}\right) \\
& =\phi\left(X_{i j} A_{j i} X_{i j}\right) .
\end{aligned}
$$

Hence it follows that $X_{i j} S X_{i j}=X_{i j} A_{j i} X_{i j}$, that is, $X_{i j}\left(S-A_{j i}\right) X_{i j}=0$. From $X_{i i}\left(S-A_{i i}\right) X_{i i}=0$, since $\mathcal{A}$ and $\mathcal{B}$ are unital algebras we have $S_{11}=A_{11}$ and $S_{22}=A_{22}$. From $X_{12}\left(S-A_{21}\right) X_{12}=0, S_{21}=A_{21}$ by condition (2). Similarly, we have $S_{12}=A_{12}$.

Lemma 2.3. $\phi$ is additive on $\mathcal{G}_{12}$.

Proof. Let $A_{11}=I_{\mathcal{A}}$, the identity element of $\mathcal{A}$ and $C_{22}=I_{\mathcal{B}}$ be that of $\mathcal{B}$. Then we have

$$
\begin{aligned}
\phi\left(A_{11}\right)+\phi\left(A_{12}+B_{12}\right)= & \phi\left(A_{11}+A_{12}+B_{12} C_{22}\right) \text { by Lemma } 2.2 \\
= & \phi\left(\left(A_{11}+C_{22}+A_{12}\right)\left(A_{11}+B_{12}\right)\left(A_{11}+C_{22}+A_{12}\right)\right) \\
= & \phi\left(A_{11}+C_{22}+A_{12}\right) \phi\left(A_{11}\right) \phi\left(A_{11}+C_{22}+A_{12}\right) \\
& +\phi\left(A_{11}+C_{22}+A_{12}\right) \phi\left(B_{12}\right) \phi\left(A_{11}+C_{22}+A_{12}\right) \\
= & \phi\left(A_{11}+A_{12}\right)+\phi\left(A_{11} B_{12} C_{22}\right) \\
= & \phi\left(A_{11}\right)+\phi\left(A_{12}\right)+\phi\left(A_{11} B_{12}\right) \\
= & \phi\left(A_{11}\right)+\phi\left(A_{12}\right)+\phi\left(B_{12}\right),
\end{aligned}
$$

from which it follows that $\phi\left(A_{12}+B_{12}\right)=\phi\left(A_{12}\right)+\phi\left(B_{12}\right)$.

Lemma 2.4. $\phi$ is additive on $\mathcal{G}_{21}$.

Proof. Let $S$ be an element of $\mathcal{G}$ such that $\phi(S)=\phi\left(A_{21}\right)+\phi\left(B_{21}\right)$. Then for every $X_{i j}$, we have

$$
\begin{aligned}
\phi\left(X_{i j} S X_{i j}\right) & =\phi\left(X_{i j}\right)\left(\phi\left(A_{21}\right)+\phi\left(B_{21}\right)\right) \phi\left(X_{i j}\right) \\
& =\phi\left(X_{i j} A_{21} X_{i j}\right)+\phi\left(X_{i j} B_{21} X_{i j}\right) .
\end{aligned}
$$

Then by Lemma 2.3, we have

$$
\begin{aligned}
\phi\left(X_{12} S X_{12}\right) & =\phi\left(X_{12} A_{21} X_{12}\right)+\phi\left(X_{12} B_{21} X_{12}\right) \\
& =\phi\left(X_{12} A_{21} X_{12}+X_{12} B_{21} X_{12}\right) .
\end{aligned}
$$


Hence we have

$$
X_{12} S X_{12}=X_{12} A_{21} X_{12}+X_{12} B_{21} X_{12} \text {. }
$$

Then by condition (2), we get $S_{21}=A_{21}+B_{21}$. By (2.1) we have

$$
\phi\left(X_{11} S X_{11}\right)=\phi\left(X_{22} S X_{22}\right)=\phi\left(X_{21} S X_{21}\right)=0 .
$$

Then it follows that $S_{11}=S_{22}=0$ and $S_{12}=0$ by condition (1), and hence

$$
\phi\left(A_{21}+B_{21}\right)=\phi\left(S_{21}\right)=\phi(S)=\phi\left(A_{21}\right)+\phi\left(B_{21}\right) .
$$

Lemma 2.5. $\phi\left(A_{11}+B_{11}\right)=\phi\left(A_{11}\right)+\phi\left(B_{11}\right)$ for every $A_{11}, B_{11} \in \mathcal{G}_{11}$.

Proof. We first claim that for every $C_{11} \in \mathcal{G}_{11}$ and $D_{12} \in \mathcal{G}_{12}$, we have that

$$
\phi\left(C_{11} D_{12}\right)=\phi\left(P_{11}\right) \phi\left(C_{11}\right) \phi\left(D_{12}\right)+\phi\left(D_{12}\right) \phi\left(C_{11}\right) \phi\left(P_{11}\right),
$$

where $P_{11}$ is the identity element of $\mathcal{A}$. By Lemma 2.2, we have

$$
\begin{aligned}
\phi\left(C_{11}\right)+\phi\left(C_{11} D_{12}\right)= & \phi\left(C_{11}+C_{11} D_{12}\right) \\
= & \phi\left(\left(P_{11}+D_{12}\right) C_{11}\left(P_{11}+D_{12}\right)\right) \\
= & \phi\left(P_{11}+D_{12}\right) \phi\left(C_{11}\right) \phi\left(P_{11}+D_{12}\right) \\
= & \left(\phi\left(P_{11}\right)+\phi\left(D_{12}\right)\right) \phi\left(C_{11}\right)\left(\phi\left(P_{11}\right)+\phi\left(D_{12}\right)\right) \\
= & \phi\left(P_{11}\right) \phi\left(C_{11}\right) \phi\left(P_{11}\right)+\phi\left(D_{12}\right) \phi\left(C_{11}\right) \phi\left(D_{12}\right) \\
& +\phi\left(P_{11}\right) \phi\left(C_{11}\right) \phi\left(D_{12}\right)+\phi\left(D_{12}\right) \phi\left(C_{11}\right) \phi\left(P_{11}\right) \\
= & \phi\left(C_{11}\right)+\phi\left(P_{11}\right) \phi\left(C_{11}\right) \phi\left(D_{12}\right)+\phi\left(D_{12}\right) \phi\left(C_{11}\right) \phi\left(P_{11}\right),
\end{aligned}
$$

from which it follows that (2.2) holds.

Now choose $S \in \mathcal{G}$ such that $\phi(S)=\phi\left(A_{11}\right)+\phi\left(B_{11}\right)$. Since for every $X_{i j} \in \mathcal{G}_{i j}$

$$
\phi\left(X_{i j} S X_{i j}\right)=\phi\left(X_{i j}\right) \phi(S) \phi\left(X_{i j}\right)=\phi\left(X_{i j} A_{11} X_{i j}\right)+\phi\left(X_{i j} B_{11} X_{i j}\right),
$$

we have

$$
\phi\left(X_{12} S X_{12}\right)=\phi\left(X_{21} S X_{21}\right)=\phi\left(X_{22} S X_{22}\right)=0 .
$$

Hence we get $S_{12}=S_{21}=S_{22}=0$ by conditions (1) and (2). We also have

$$
\begin{aligned}
\phi\left(S_{11} X_{12}\right) & =\phi\left(P_{11}\right) \phi\left(S_{11}\right) \phi\left(X_{12}\right)+\phi\left(X_{12}\right) \phi\left(S_{11}\right) \phi\left(P_{11}\right) \\
& =\phi\left(P_{11}\right)\left(\phi\left(A_{11}\right)+\phi\left(B_{11}\right)\right) \phi\left(X_{12}\right)+\phi\left(X_{12}\right)\left(\phi\left(A_{11}\right)+\phi\left(B_{11}\right)\right) \phi\left(P_{11}\right) \\
& =\phi\left(A_{11} X_{12}\right)+\phi\left(B_{11} X_{12}\right) \\
& =\phi\left(A_{11} X_{12}+B_{11} X_{12}\right),
\end{aligned}
$$

where the first and third equations hold by (2.2) and the last equation comes by the additivity of $\phi$ on $\mathcal{G}_{12}$. Then we get $S_{11}=A_{11}+B_{11}$ by condition (3), and hence

$$
\phi\left(A_{11}+B_{11}\right)=\phi\left(A_{11}\right)+\phi\left(B_{11}\right) .
$$

Lemma 2.6. $\phi$ is additive on $\mathcal{G}_{22}$. 
Proof. Let $A_{22}$ and $B_{22}$ be elements of $\mathcal{G}_{22}$ and $S \in \mathcal{G}$ be an element such that $\phi(S)=\phi\left(A_{22}\right)+\phi\left(B_{22}\right)$. Since for every $X_{i j} \in \mathcal{G}_{i j}$

$$
\phi\left(X_{i j} S X_{i j}\right)=\phi\left(X_{i j}\right) \phi(S) \phi\left(X_{i j}\right)=\phi\left(X_{i j} A_{22} X_{i j}\right)+\phi\left(X_{i j} B_{22} X_{i j}\right),
$$

we have

$$
\phi\left(X_{11} S X_{11}\right)=\phi\left(X_{11}\right) \phi\left(A_{22}\right) \phi\left(X_{11}\right)+\phi\left(X_{11}\right) \phi\left(B_{22}\right) \phi\left(X_{11}\right)=0 .
$$

Hence $X_{11} S X_{11}=0$ and $X_{11} S_{11} X_{11}=0$. Then it follows that $S_{11}=0$. Since $\phi\left(X_{12} S X_{12}\right)=0$ by $(2.3)$, we have $X_{12} S X_{12}=0$ and hence $S_{21}=0$. Similarly, $S_{12}=0$. Hence

$$
\phi\left(S_{22}\right)=\phi\left(A_{22}\right)+\phi\left(B_{22}\right) .
$$

Considering, for every $X_{12}, Y_{12} \in \mathcal{G}_{12}$

$$
\begin{aligned}
\phi\left(X_{12} S_{22} Y_{21}\right) & =\phi\left(\left(X_{12}+Y_{21}\right) S_{22}\left(X_{12}+Y_{21}\right)\right) \\
& =\phi\left(X_{12}+Y_{21}\right) \phi\left(S_{22}\right) \phi\left(X_{12}+Y_{21}\right) \\
& =\phi\left(X_{12}+Y_{21}\right)\left(\phi\left(A_{22}\right)+\phi\left(B_{22}\right)\right) \phi\left(X_{12}+Y_{21}\right) \\
& =\phi\left(\left(X_{12}+Y_{21}\right) A_{22}\left(X_{12}+Y_{21}\right)\right)+\phi\left(\left(X_{12}+Y_{21}\right) B_{22}\left(X_{12}+Y_{21}\right)\right) \\
& =\phi\left(X_{12} A_{22} Y_{21}\right)+\phi\left(X_{12} B_{22} Y_{21}\right) \\
& =\phi\left(X_{12} A_{22} Y_{21}+X_{12} B_{22} Y_{21}\right)
\end{aligned}
$$

we have

$$
X_{12} S_{22} Y_{21}=X_{12} A_{22} Y_{21}+X_{12} B_{22} Y_{21} \text {. }
$$

Therefore by condition (4), it follows that $S_{22}=A_{22}+B_{22}$, and hence

$$
\phi\left(A_{22}+B_{22}\right)=\phi\left(A_{22}\right)+\phi\left(B_{22}\right) .
$$

We now prove our main result.

Proof of Theorem 1.1. Let $A=A_{11}+A_{12}+A_{21}+A_{22}$ and $B=B_{11}+B_{12}+$ $B_{21}+B_{22}$ be elements of $\mathcal{G}$. Then by Lemmas $2.2,2.3,2.4,2.5$, and 2.6, we have

$$
\begin{aligned}
\phi(A+B) & =\phi\left(A_{11}+B_{11}+A_{12}+B_{12}+A_{21}+B_{21}+A_{22}+B_{22}\right) \\
& =\phi\left(A_{11}+B_{11}\right)+\phi\left(A_{12}+B_{12}\right)+\phi\left(A_{21}+B_{21}\right)+\phi\left(A_{22}+B_{22}\right) \\
& =\phi\left(A_{11}\right)+\phi\left(B_{11}\right)+\phi\left(A_{12}\right)+\phi\left(B_{12}\right) \\
& +\phi\left(A_{21}\right)+\phi\left(B_{21}\right)+\phi\left(A_{22}\right)+\phi\left(B_{22}\right) \\
& =\phi\left(A_{11}\right)+\phi\left(A_{12}\right)+\phi\left(A_{21}\right)+\phi\left(A_{22}\right) \\
& +\phi\left(B_{11}+\phi\left(B_{12}\right)+\phi\left(B_{21}\right)+\phi\left(B_{22}\right)\right. \\
& =\phi(A)+\phi(B) .
\end{aligned}
$$

This completes the proof.

Acknowledgements. The authors thank the referees for the very through reading of the article and many helpful comments. 


\section{References}

[1] X. Cheng and W. Jing, Additivity of maps on triangular algebras, Electron. J. Linear Algebra 17 (2008), 597-615.

[2] I. Hakeda, Additivity of Jordan *-maps on $A W^{*}$-algebras, Proc. Amer. Math. Soc. 96 (1986), no. 3, 413-420.

[3] J. Hakeda and K. Saito, Additivity of Jordan *-maps between operator algebras, J. Math. Soc. Japan 38 (1986), no. 3, 403-408.

[4] P. Ji, Jordan maps on triangular algebras, Linear Algebra Appl. 426 (2007), no. 1, 190-198.

[5] P. Ji, R. Liu, and Y. Zhao, Nonlinear Lie triple derivations of triangular algebras, Linear Multilinear Algebra 60 (2012), no. 10, 1155-1164.

[6] W. Jing, Additivity of Jordan elementary maps on rings, arXiv:0706.0488v1 [math.RA] 4 Jun 2007.

[7] B. Kuzma, Jordan triple product homomorphisms, Monatsh. Math. 149 (2006), no. 2, 119-128.

[8] P. Li and W. Jing, Jordan elementary maps on rings, Linear Algebra Appl. 382 (2004), $237-245$.

[9] P. Li and F. Lu, Additivity of Jordan elementary maps on nest algebras, Linear Algebra Appl. 400 (2005), 327-338.

[10] Y. Li and Z. Xiao, Additivity of maps on generalized matrix algebras, Electron. J. Linear Algebra 22 (2011), 743-757.

[11] Z. Ling and F. Lu, Jordan maps of nest algebras, Linear Algebra Appl. 387 (2004), 361-368.

[12] F. Lu, Jordan triple maps, Linear Algebra Appl. 375 (2003), 311-317.

[13] Jordan maps on associative algebras, Comm. Algebra 31 (2003), no. 5, 22732286

[14] Multiplicative mappings of operator algebras, Linear Algebra Appl. 347 (2002), $283-291$.

[15] Additivity of Jordan maps on standard operator algebras, Linear Algebra Appl. 357 (2002), 123-131.

[16] A. J. C. Martín and C. M. González, The Banach-Lie group of Lie triple automorphisms of an $H^{*}$-algebras, Acta Math. Sci. Ser. B Engl. Ed. 30 (2010), no. 4, 1219-1226.

[17] A linear approach to Lie triple automorphisms of $H^{*}$-algebras, J. Korean Math. Soc. 48 (2011), no. 1, 117-132.

[18] W. S. Martindale III, When are multiplicative mappings additive?, Proc. Amer. Math. Soc. 21 (1969), 695-698.

[19] L. Molnár, On isomorphisms of standard operator algebras, Studia Math. 142 (2000), no. 3, 295-302.

[20] L. Molnár and P. Šemrl, Elementary operators on standard algebras, Linear Multilinear Algebra 50 (2002), no. 4, 315-319.

[21] P. Šemrl, Isomorphisms of standard operator algebras, Proc. Amer. Math. Soc. 123 (1995), no. 6, 1851-1885.

[22] Z. Xiao and F. Wei, Commuting mappings of generalized matrix algebras, Linear Algebra Appl. 433 (2010), no. 11-12, 2178-2197.

[23] Lie triple derivations of triangular algebras, Linear Algebra Appl. 437 (2012), no. $5,1234-1249$.

SANG OG KIM

Department of Mathematics

HALLYM UNIVERSITY

Chuncheon 200-702, Korea

E-mail address: sokim@hallym.ac.kr 
Choonkil Park

Research Institute for Natural Sciences

HANYANG UNIVERSITY

SEOUl 133-791, KoreA

E-mail address: baak@hanyang.ac.kr 